\title{
Acute Colonic Pseudo-Obstruction: Local Experience
}

\section{Shawki MK Sharouda, ${ }^{1}$ MD; HosamE.N Ibrahim, ${ }^{2}$ MD; Gehan G Ali, ${ }^{3}$ MD; Abeer SM Mohamed, ${ }^{4}$ MD; Mohammed Taema, ${ }^{5}$ MD.}

\author{
1) General Surgery Department, Faculty of Medicine, Ain Shams University, \\ Egypt. \\ 2) Internal Medicine Department, Faculty of Medicine, Ain Shams University, \\ Egypt. \\ 3) Radiology Department, Faculty of Medicine, Ain Shams University, Egypt. \\ 4) Radiology Department, Faculty of Medicine, Tanta University, Egypt. \\ 5) Obstetrics and Gynecology Department, Faculty of Medicine, Ain Shams \\ University, Egypt.
}

Background: In countries like Saudi Arabia where multiple nationalities are present in the same place, the rare and uncommon diseases are to be considered. Acute colonic pseudoobstruction (ACPO), also known as Ogilvie's syndrome is an uncommon condition that occasionally develops in hospitalized patients with serious underlying illness. It is characterized by the signs, symptoms and radiological evidence of a large-bowel obstruction, but without mechanical cause. Early recognition and diagnosis of this condition allows for treatment prior to bowel perforation and requisite abdominal surgery. The aim of this study was to review our experience in assessing the circumstances, the clinical, and methods of management of acute colonic pseudo-obstruction.

Patients and methods: From July 2005 to November 2015, all patients with proven acute colonic pseudo-obstruction were identified in the retrospective way at two private hospitals in Jeddah, KSA. 16 patients affected by pseudo-obstruction of the colon were reviewed. Acute dilatation of the colon without organic obstruction was the inclusion criterion for the study.

Result: In the ten-year period, 16 patients had symptoms, signs and radiological appearance ACPO. Their average age was 49 years. 5 patients were post Caesarean section, 4 patients had orthopaedic procedures, one patient was post hysterectomy and 6 patients with different medical diseases. 14 patients were treated by conservative, pharmacological or colonoscopic decompression while two patients required laparotomy for perforated cecum duo to delayed diagnosis.

Conclusions: Acute colonic pseudo-obstruction (Ogilvie's syndrome) is a rare condition. It should be included in the differential diagnosis of postoperative abdominal distension and pain. Decisions about the need for pharmacological therapy, colonoscopy, or surgery should be individualized and based on the patient's clinical status. Early diagnosis can prevent major morbidity and mortality due to bowel ischemia and perforation.

Key words: Acute colonic pseudo-obstruction, Ogilvie's syndrome, colonoscopic decompression, cecostomy.

\section{Introduction:}

Acute colonic pseudo-obstruction is a critical medical condition which is poorly understood and an uncommon syndrome.
It is characterized by signs of large bowel obstruction (abdominal distension and colonic dilatation) without a mechanical cause. ${ }^{1}$ This condition usually develops in 
hospitalized patients and is associated with a range of medical and surgical conditions. If inappropriately managed, it may result in ischemic necrosis and colonic perforation, with a mortality rate as high as fifty percent. $^{2}$ An imbalance in the autonomic innervations (sympathetic over activity and parasympathetic suppression) has been thought to be the patho-physiological factor in the causation of this condition.1,3

In 1948, Ogilvie first described two patients with metastatic disease to the celiac ganglia who were examined for signs and symptoms of obstruction, despite normal barium enema studies. Ogilvie concluded that the nerve supply to the colon was affected by these tumors and the neurologic dysfunction led to "pseudo-obstruction". ${ }^{1}$ Dudley et $\mathrm{al}^{4}$ in 1958 recognized the obstruction to be due to functional rather than mechanical causes and the name of acute colonic pseudo-obstruction first appeared in the literature by Nanni et al. in $1982.4,5$

In spite of multiple case reports and series, the actual mechanism of colonic dilatation in the absence of obstruction remains unclear. Common medical conditions associated with Ogilvie's syndrome include; recent surgery, general anaesthesia, sepsis, electrolyte abnormalities, certain medications (i.e. opioids, antidepressants), cardiac diseases, respiratory failure, and neurologic dysfunction. ${ }^{6}$

Spira et $\mathrm{al}^{3}$ in 1976 noted that the most common event in their series was caesarean section (35\%). Urologic surgery is the second most common associated procedure in men. In another series involving 400 patients with acute colonic pseudo-obstruction who had antecedent surgery, 19\% had obstetric, gynaecologic, or pelvic operation. ${ }^{7}$

Despite the accurate description and increase awareness of this condition, its diagnosis remains difficult, and is sometimes delayed. So, early detection and appropriate treatment are essential to minimizing morbidity and mortality. 8

\section{Patients and methods:}

From July 2005 to November 2015, all patients with acute colonic pseudo-obstruction from inpatient medical and surgical wards of two private hospitals in Jeddah, KSA were collected in this study. Patients' details in relation to their symptoms (which include pain associated with abdominal distension, nausea/vomiting and constipation), diagnosis and management were recorded. All had clinical and radiological features of Ogilvie's syndrome. Acute colonic pseudo-obstruction was defined as marked colonic distention in the absence of mechanical obstruction. To be eligible for the study, patients had to have a cecal diameter of at least $9 \mathrm{~cm}$ on plain radiographs Figure (1,2).

Mechanical obstruction was ruled out by the finding of air throughout all colonic segments on plain abdominal $\mathrm{x}$-ray or by radiographic contrast enemas if air was not demonstrable in the rectosigmoid colon

Conservative treatment included administering nothing by mouth, nasogastric suction, intravenous fluid, correction of electrolyte imbalances and frequent changes in the patient's position. The length of time before conservative treatment was begun varied from two to six days postoperatively.

Patients who did not improved on conservative therapy were candidates for further interventions included medical treatment and endoscopic decompression.

Oral erythromycin at a dose of $500 \mathrm{mg}$, three times per day for three to four days was given. Patients received neostigmine in the dose of $2 \mathrm{mg}$ intravenously over a period of 3-5 minutes with continuous ECG monitoring and atropine available at bedside.

Laxatives were avoided, and medications that can affect colonic motility, such as opiates, anticholinergic and calcium channel antagonists drugs were discontinued when possible.

Observation of the patient clinically, abdominal girth measurement, and plain x-ray abdomen were done daily. CT abdomen was done in selected patients according to their conditions Figure $(3,4)$.

Exclusion criteria included history of colon cancer, partial colonic resection, active gastrointestinal bleeding or pregnancy. 
The study protocol was approved by the local ethics committees and written informed consent forms were obtained from all subjects before their entrance into the study.

\section{Results:}

Over the period of 10 years, 16 patients (10 female and 6 male) had the criteria for a diagnosis of acute colonic pseudo-obstruction (ACPO). The mean age of the patient was 49 years (range 31-90 years).

All patients had acute abdominal distention associated with one or more of the following symptoms: abdominal pain, nausea, vomiting and constipation.

5 patients had caesarean section, 4 patients had orthopaedic procedures (Hip replacement or fractures), two had cardiovascular disease, hysterectomy, liver cirrhosis, sepsis, renal failure and pneumonia with respiratory failure, in one patient each.

Two patients out of 16 required laparotomy for perforated cecum due to delayed diagnosis.

Five patients were improved on conservative treatment alone and colonic distention gradually resolved over the next 3 to 4 days, while it was failed in other nine patients.

Four patients were started on oral erythromycin, two patients had a beneficial response to it, but recurrence occurred in one of them. The other 2 patients had no response to erythromycin.

4 out of the remaining 5 patients, in addition to the two who failed response to oral erythromycin received neostigmine intravenously. One patient did not receive neostigmine due to his general condition. Five patients got improved on neostigmine while one did not Figure (5).

Three patients had been treated successfully by colonoscopy decompression; however, one of them needed twice decompression.

The two cases that had cecal perforation presented as following:

A 90 year old female, presented to the emergency department with history of falling leading to right femur fracture. She was admitted in orthopaedic department where conservative management and external traction were planned. After 4 days she developed abdominal discomfort, associated with nausea and constipation.

Her physical examination was remarkable for moderately abdominal distention; bowel sounds were present in all quadrants. Her abdomen was soft and not tender to palpation, without rebound or guarding. The general vital signs were within normal. Conservative treatment was planned by her physician. 3 days later, in addition to abdominal distention, patient started to complain of abdominal pain, nausea and low grade fever. Surgical consultation was done as there was no improvement of her condition on conservative management. Physical examination showed general appearance of a frail, malnourished elderly woman with decreased mental status as well as tachycardia and low grade fever.

Abdominal examination showed a massive distended abdomen, with mild tenderness and rigidity. An abdominal x-ray was ordered to rule out a bowel obstruction versus perforation. It revealed pneumoperitoneum and a dilated colon. An emergency laparotomy was performed. She had a perforation of her cecum with pus and straw-coloured fluid in her abdomen. Peritoneal toilet and cecostomy were done. Patient passed stool from cecostomy after 6 days and was discharged 3 weeks after surgery in good condition.

A 33 year-old female third gravida had an elective Caesarean section for prolonged second stage of labour. Patient started oral feeding in the second postoperative day. She developed abdominal distension and nausea from the third post-operative day. Plain x-ray revealed dilated large bowel with no signs of obstruction. So, conservative treatment was planned by her physician. 2 days later, patient started to complain of severe abdominal pain, nausea and vomiting with fever, tachycardia and tachypnea. Surgical consultation was done where the patient looked toxic, with abdominal distention, tenderness and rigidity all over the abdomen. Abdominal x-ray showed multiple fluid levels with air under diaphragm. Emergency laparotomy showed markedly distended colon with perforated cecum (2 small adjacent perforations). 
Peritoneal toilet and tube cecostomy were done. Post-operative recovery was uneventful and patient went home 7 days after surgery. Patient developed incisional hernia, which was repaired later.

\section{Discussion:}

The evaluation of a patient with acute colonic distension is challenging and must always be considered a medical emergency regardless of the patient's age. Acute colonic pseudo-obstruction is a well-recognized syndrome for which innovative new treatments have recently been introduced. ${ }^{9}$ Acute colonic pseudo-obstruction is defined as acute colonic dilatation in the absence of obvious colonic disease or mechanical obstruction. This definition excludes toxic colitis, which occurs in the setting of severe colitis secondary to inflammatory bowel disease or infection. ${ }^{10}$ The mortality rate in ACPO is $15 \%$ with early appropriate care but increases to $36 \%$ if the patient progresses to colonic ischemia and perforation. Pharmacologic and endoscopic interventions have been proposed to prevent this disease progression and reduce mortality. ${ }^{7}$

Pathology of acute intestinal obstruction without mechanical obstruction is not clear. The initial theory to explain the acute colonic pseudo-obstruction was an imbalance in the activity of autonomic nervous system with parasympathetic overactivity leading to dilation of the colon. ${ }^{1}$ Because the vagus innervates the large bowel to the splenic flexure and the sacral parasympathetic nerves innervate the left colon, another theory proposed that transient impairment of the sacral parasympathetic nerves may cause atony of the distal large bowel,with progressive bowel dilatation entrapping large amounts of air and fluid stools within the lumen. These events result in marked dilatation of the proximal and transverse colon and in turn responsible for functional obstruction. ${ }^{11}$ However, current evidence favors a relatively increased sympathetic tone and/or decreased parasympathetic tone leading to a functionally obstructing distal colon and a relaxed proximal colon.
The evidence in favor of this theory is the association of ACPO with several diseases causing a disturbance in the autonomic flow to the gut and a remarkable response to pharmacologic therapy. ${ }^{12}$

The pathophysiologic mechanisms underlying Ogilvie's syndrome are still poorly understood. The syndrome is associated with a vast array of clinical conditions and occurs in patients with peritonitis, gram-negative sepsis, retroperitoneal haemorrhage, myocardial infarction, pancreatitis, alcohol abuse, and pneumonia. ${ }^{13}$ Surgery has preceded Ogilvie's syndrome in $50 \%$ to $60 \%$ of patients which including orthopaedic procedures, coronary bypass grafts, abdominal and pelvic surgery. ${ }^{14}$ Nine patients $(56 \%)$ in this study [5 post caesarean section, 3 orthopaedic procedures and one hysterectomy] was preceded by surgery.

Spira et $\mathrm{al}^{3}$ found that caesarean section was the most common associated surgery and this was confirmed by Vanek et al, ${ }^{7}$ while not agreed by others. ${ }^{13,14}$

The interval from operation to diagnosis of Ogilvie's syndrome is typically 3 to 5 days. Tenderness is mild or absent and much less than one would expect for the degree of abdominal or cecal enlargement. Nausea and vomiting are not consistently present. Bowel sounds may be normal, hyperactive, or hypoactive. Mild leukocytosis is common. More than $20 \%$ of patients exhibit hypocalcaemia, hyponatraemia, and alkalosis. Severe abdominal pain or a marked increase in leukocytosis is warning signs for impending or actual perforation. ${ }^{13}$

Ogilvie's syndrome appears to be more common in men and in patients over the age of 60 years,7,8 however, this was not similar to those found in our study, may be as nearly third of our patients were female in childbearing period.

The main criteria for the diagnosis are marked distension of the cecum on abdominal radiographs and absence of mechanical obstruction. According to the law of LaPlace, the pressure required to stretch the walls of a hollow viscus decreases inversely to the diameter. The tensile strength of the cecum 


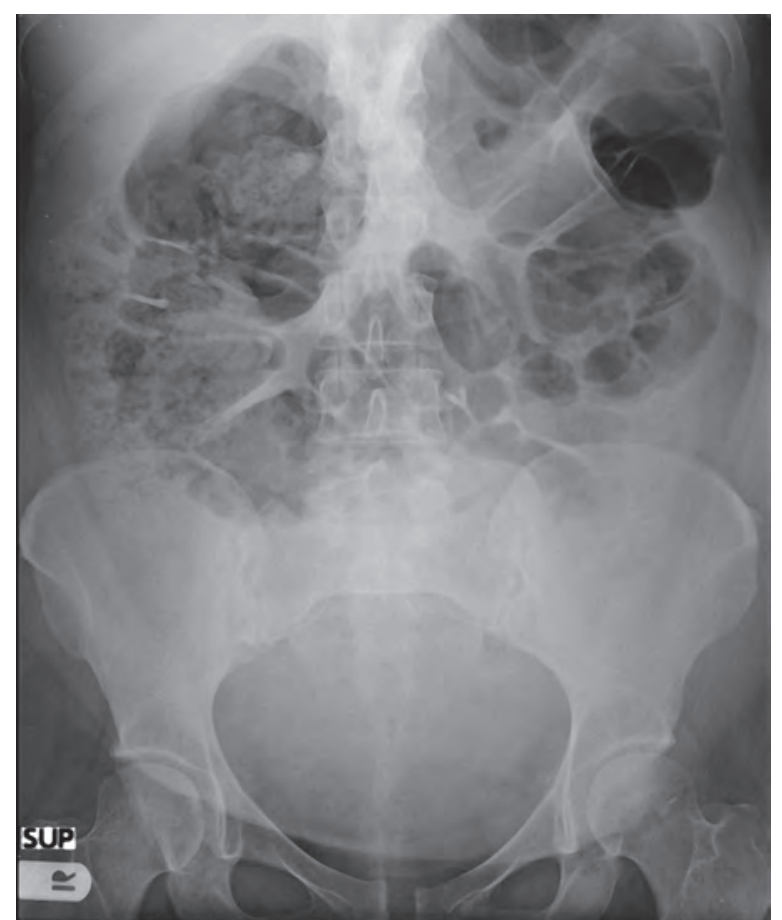

Figure (1): Plain x-ray abdomen showed dilated cecum and large bowel.

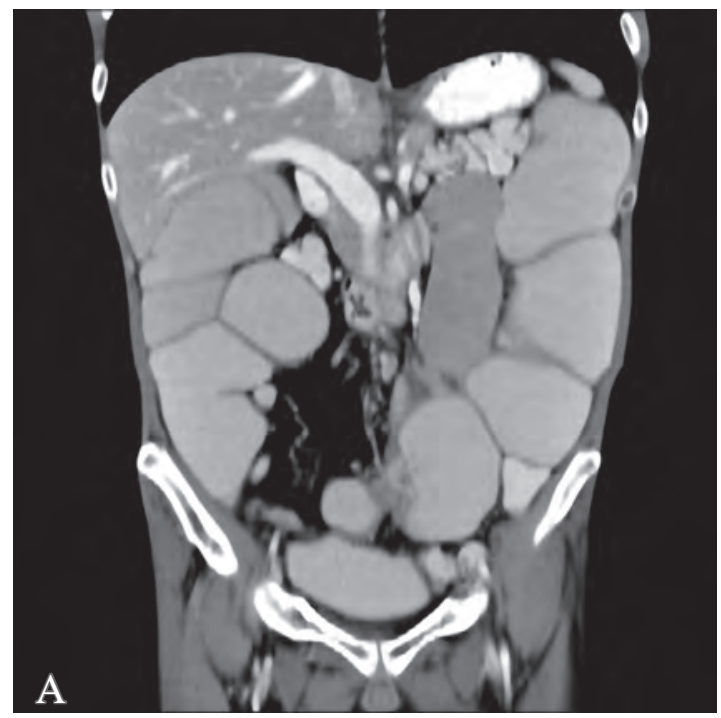

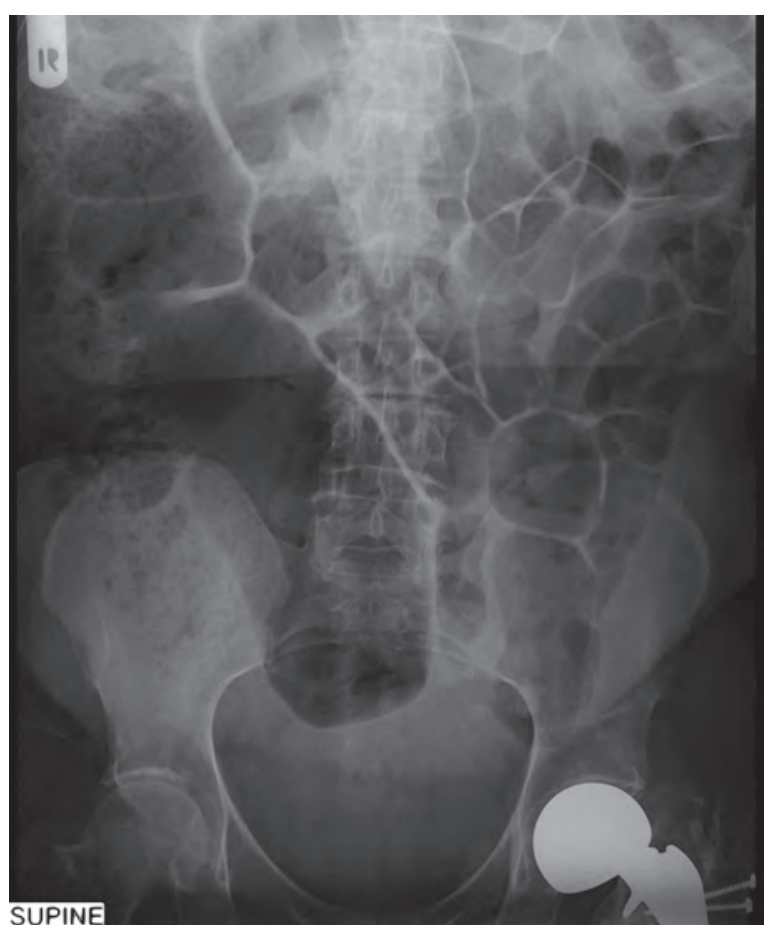

Figure (2): Plain x-ray abdomen for patient with left hip total arthroplasty showed dilated cecum and large bowel.

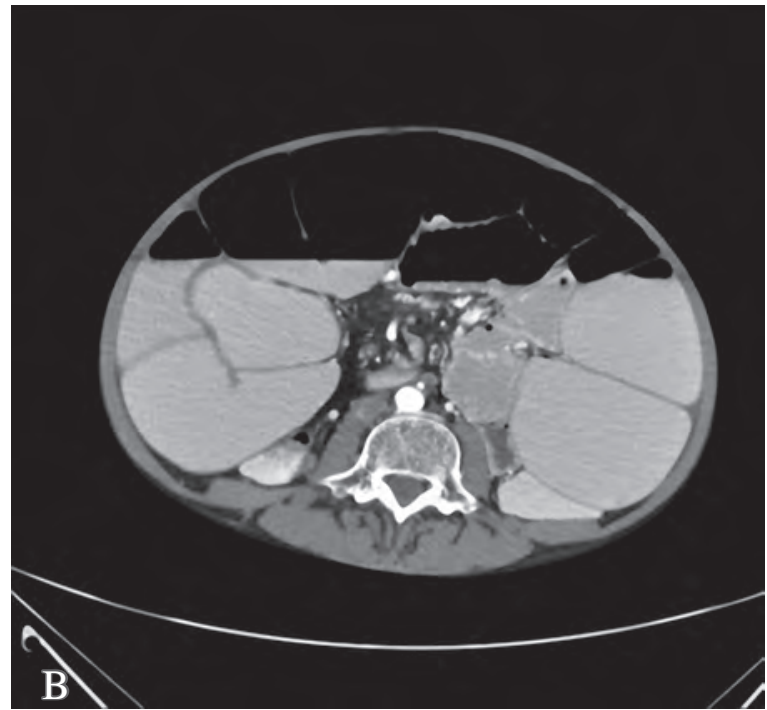

Figure (3): (A) axial \& (B) coronal CT cuts of the abdomen show dilated colon from cecum to rectum with no definite mechanical obstruction seen.

is exceeded earlier than in the remainder of the colon because it has the larger diameter. Progressive distension leads to ischemia and perforation. While the risk of perforation for cecal dilatation is undefined, it occurs uncommonly. 15 Perforation from cecal dilatation is rare, but it is associated with mortality rates approaching $50 \% .{ }^{16}$

The actual diameter at which perforation of the cecum occurs remains debatable. Daviset al $^{17}$ reported impending cecal perforation when the measurement exceeded $9 \mathrm{~cm}$, while Vanek et $\mathrm{al}^{7}$ review of 400 cases showed no perforation or ischemic changes until the diameter of the cecum exceeded $12 \mathrm{~cm}$.

Because of the broad overlap in cecal diameters between patients in whom acute colonic pseudo-obstruction resolves and those in whom perforation occurs, some have suggested that the duration of dilatation 


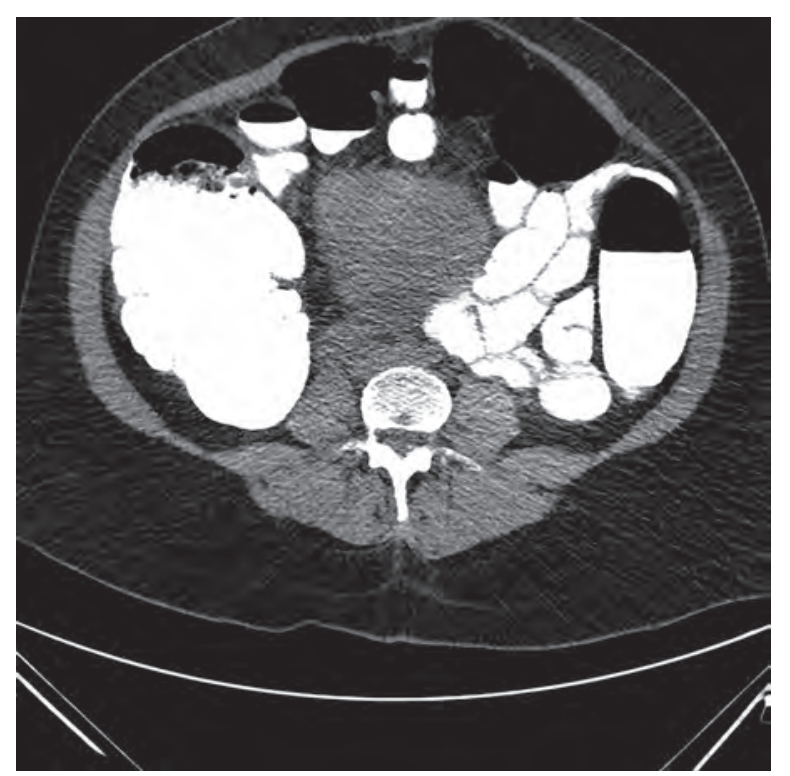

Figure (4): CT with oral contrast revealed dilated cecum and large bowel.

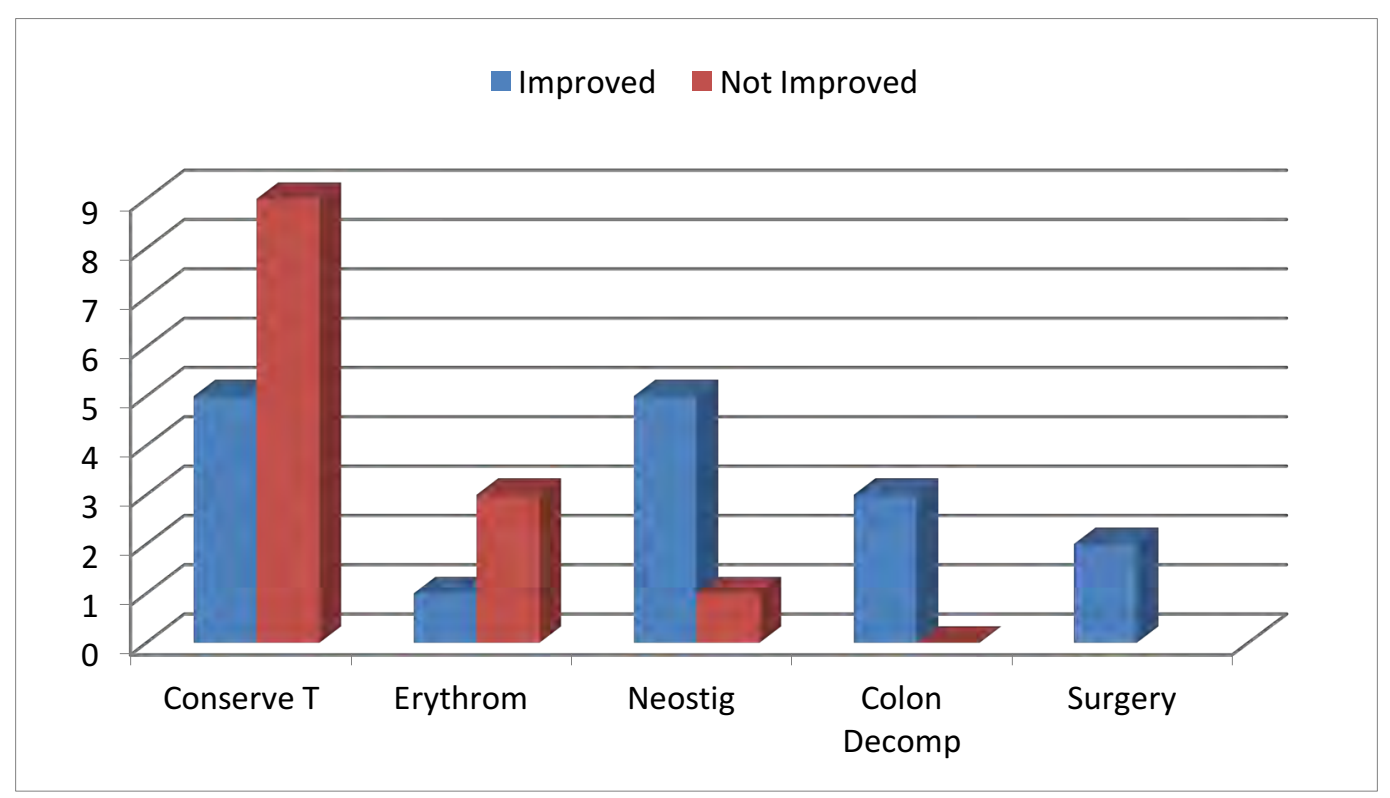

Figure (5): Results of ACPO treatment. \{Conserve $T=$ Conservative Treatment, Erythrom = Erythromycin, Neostig = Neostigmine, Colon Decomp $=$ Colonoscopic Decompression $\}$.

[probably more than 6 days] may be a more important risk factor. ${ }^{18}$

Once mechanical obstruction has been ruled out, management is initially conservative and includes, cessation of feeding by mouth, placing a nasogastric tube gently suction, insertion of a rectal probe if the distension reaches the sigmoid or rectum and changing the patient's position in order to stimulate the emission of gas and stool. In addition to correction of metabolic disturbances and discontinuation of medications that may decrease colonic motility, and treatment of underlying medical conditions. ${ }^{19,20}$ The durations of conservative management ranging from 3 days to 6 days if clinical signs of perforation were absent, and cecal diameters were $\leq 9 \mathrm{~cm} .{ }^{13,21}$ Abdominal radiographs should be repeated every 12 to 24 hours to monitor changes in cecal diameter. If symptoms persist or worsen, or if the colonic diameter increases or remains, pharmacologic decompression of the colon is generally recommended. ${ }^{20}$

Several pharmacologic approaches have been attempted in the treatment of ACPO. 
The erythromycin a macrolide antibiotic is known to stimulate gastric and small bowel motor activity as its prokinetic effect is ascribed to the binding to the motilin receptor. ${ }^{22}$ The efficacy of erythromycin in Ogilvie's syndrome is documented in some series, ${ }^{23-25}$ however, it is not used on wide scale recently.

Neostigmine, is an anticholinesterase (parasympathomimetic), and acts to increase the acetylcholine concentration at the synapses of the enteric nervous system resulting in increase in colonic motility. Patients eligible for Neostigmine therapy must not be mechanically obstructed, have a baseline heart rate greater than 60 beats/min, or have a systolic blood pressure greater than $90 \mathrm{~mm} \mathrm{Hg}$. Other exclusion criteria include signs of bowel ischemia or perforation, active gastrointestinal bleeding, renal failure, recent myocardial infarction and active bronchospasm requiring medication. ${ }^{26,27}$ Patients receiving neostigmine should be monitored for cardiac arrhythmia and atropine must be available at the bedside to treat severe bradycardia. ${ }^{28}$

Ponec et $\mathrm{al}^{26}$ performed a randomized trial of neostigmine for the treatment of patients with Ogilvie's syndrome. 91\% of patients who were treated with intravenous neostigmine had prompt passage of flatus or stools and reduced abdominal distension, as compared with other patients who received placebo.

In other recent meta-analysis study, the neostigmine effectiveness to resolve ACPO with only one dose averaged was $89.2 \%$ (ranging from 84.6 to $95.2 \%{ }^{29}$ These results are similar to those found in our study as the success rate after neostigmine was $83 \%$.

Use of other compounds (muscarinic receptor agonists, neurotrophins, somatostatin analogs and 5-HT4 receptor agonist) is limited by the occurrence of significant side effects or the lack of clinical data on colonic motility. 30

Should the patient fail to respond to or be unsuitable for medical therapy, colonoscopic orsurgical decompression should beattempted. Colonoscopic decompression of ACPO was first successful in 1977. ${ }^{31}$ It is successful in approximately $70 \%$ to $95 \%$ of patients with Ogilvie's syndrome if the hepatic flexure can be reached. ${ }^{32,33}$ Dilatation will recur in $20 \%$ to $40 \%$ of patients, requiring a repeated colonoscopy. ${ }^{2}$ Recurrent dilatation may be decreased by the placement of a drainage tube into the right side of the colon at the time of colonoscopy. ${ }^{34}$ All these results are nearly consistent with ours; however, we had only 3 cases did colonoscopic decompression.

Surgical intervention is rarely necessary and it is reserved for patients with ischemia, perforation, or after failure of pharmacologic or endoscopic therapy. Ogilvie's syndrome is one of the few indications for cecostomy due to the relative simplicity of the procedure and the prompt decompression. Surgical decompression may be successful with a tube cecostomy, surgical cecostomy, Colonoscopically guided cecostomy, 35 percutaneous approach with CT guidance, ${ }^{36}$ right hemicolectomy to total abdominal colectomy.7,10,13 Even in the absence of perforation, surgery carries a significant mortality rate. In one retrospective series of 179 patients undergoing surgery for ACPO, the morbidity and mortality rates were $6 \%$ and $30 \%$, respectively, where mortality was more common in patients with either ischemic or perforation. ${ }^{7}$ However, in another series, the authors attributed the high mortality rate to the co-morbidities and the underlying acute conditions in this group of patients. ${ }^{13}$

\section{Conclusion:}

Acute colonic pseudo-obstruction (Ogilvie's syndrome) is a rare but potentially dangerous condition in hospitalized medical and surgical patients, resulting due to parasympathetic suppression. The condition can be diagnosed early because of its classical presentation (both clinical and radiographic) makes it amenable to an early treatment, which can prevent or minimize its complications. Appropriate management includes conservative, pharmacologic therapy, or endoscopic decompression which has a potential to reduce the need of critical surgery in those patients. Obstetricians and 
orthopedic surgeons should be cognizant of this condition in the patient whose abdomen becomes distended postoperatively with early consultation of a gastroenterologist and general surgeon to avoid delayed diagnosis.

The limitations of our study are its retrospective nature, few number of patients and had no standardized protocol was predefined to manage those patients.

\section{Conflict of Interest:}

The authors declare no conflict of interest.

\section{Reference:}

1- Ogilvie H: Large-intestine colic due to sympathetic deprivation: A new clinical syndrome. BMJ 1948; 2: 671-673.

2- Rex DK: Colonoscopy, and acute colonic pseudo-obstruction. Gastrointest Endosc Clin N Am 1997; 7: 499-508.

3- Spira IA, Rodrigues R, Wolff WI: Pseudoobstruction of the colon. Am J Gastroentrol 1976; 65: 397-408.

4- Dudley H AF, Sinclair IS, McLaren IF, et al: Intestinal pseudo-obstruction. $J$ R Coll Surg Edinb 1958; 3(3): 206-217.

5- Nanni G, Garbini A, Luchetti P, et al: Ogilvie's syndrome (acute colonic pseudoobstruction): Review of the literature (October 1948 to March 1980) and report of four additional cases. Dis Colon Rectum 1982; 25(2): 157-166.

6- Maloney $\mathrm{N}$, Vargas $\mathrm{H}$ : Acute intestinal pseudo-obstruction (Ogilvie's syndrome). Clin Colon Rectal Surg 2005; 18(2): 96-101.

7- Vanek VW, Al-Salti M: Acute pseudoobstruction of the colon (Ogilvie's syndrome): An analysis of 400 cases. Dis Colon Rectum 1986: 19: 203-210.

8- Saunders MD, Kimmey MB: Systematic review: Acute colonic pseudo-obstruction. Aliment Pharmacol Ther 2005; 22(10): 917-925.

9- Weinstock LB, Chang AC: Methyl naltrexone for treatment of acute colonic pseudoobstruction. J Clin Gastroenterol 2011; 45(10): 883-884.

10- Eisen GM, Baron TH, Dominitz JA, et al: Acute colonic pseudo-obstruction. Gastrointest Endosc 2002; 56(6): 789-792.

11- Bachulis BL, Smith PE: Pseudo-obstruction of the colon. Am J Surg 1978; 136: 66-72.

12- Durai R: Colonic pseudo-obstruction.
Singapore Med J 2009; 50(3): 237-244.

13- Tenofsky PL, Beamer L, Smith RS: Ogilvie syndrome as a postoperative complication. Arch Surg 2000; 135: 682-686.

14- Jetmore $\mathrm{AB}$, Timmcke AE, Gathright JB, et al: Ogilvie's syndrome: Colonoscopic decompression and analysis of predisposing factors. Dis Colon Rectum 1992; 35: 1135-1142.

15- Kaiser AM: Ogilvie transition to colonic perforation. Am J Surg 2010; 200(1): 15-16.

16- Reece EA, Petrie RH, Hutcherson H: Ogilvie's syndrome in the post-cesarean section patient. Am J Obstet Gynecol 1996; 175: 849-850.

17- Davis L, Lowman RM: An evaluation of cecal size in impending perforation of the cecum. Surg Gynecol Obstet 1956; 103(6): 711-718.

18- Saunders MD: Acute colonic pseudoobstruction. Best Pract Res Clin Gastroenterol 2007; 21: 671-687.

19- Sloyer AF, Panella VS, Demas BE, et al: Ogilvie's syndrome: Successful management without colonoscopy. Dig Dis Sci 1988; 33: 1391-1396

20- Guidelines of the American Society for Gastrointestinal Endoscopy. The role of endoscopy in the management of patients with known and suspected colonic obstruction and pseudo-obstruction. Gastrointestinal Endoscopy 2010; 71: 669-681.

21- Alwan MH, van RijAM: Acute colonic pseudo-obstruction. Aust N Z J Surg 1998; 68: 129-132.

22- Peeters TL: Erythromycin and other macrolides as prokinetic agents. Gastroenterology 1993; 105: 1886-1899.

23- Armstrong DN, Ballantyne GH, Modlin IM: Erythromycin for reflex ileus in Ogilvie's syndrome. Lancet 1991; 337(8737): 378.

24- Bonacini M, Smith OJ, Pritchard T: Erythromycin as therapy for acute colonic pseudo-obstruction (Ogilvie's syndrome). $J$ Clin Gastroenterol 1991; 13: 475-476.

25- Rovira A, Lopez A, Cambray C, Gimeno C: Acute colonic pseudo-obstruction (Ogilvie's syndrome) treated with erythromycin. Intensive Care Med 1997; 23: 798-798.

26- Ponec RJ, Saunders MD, Kimmey MB: Neostigmine for the treatment of acute colonic pseudo-obstruction. $N$ Engl J Med 1999; 341: 137-141.

27- Abeyta BJ, Albrecht RM, Schermer CR: Retrospective study of neostigmine for 
the treatment of acute colonic pseudoobstruction. Am Surg 2001; 67: 265-268.

28- De Giorgio R, Knowles CH: Acute colonic pseudo-obstruction. Br J Surg 2009; 96(3): 229-239.

29- Valle RG, Godoy FL: Neostigmine for acute colonic pseudo-obstruction: A meta-analysis. Ann Med Surg (Lond) 2014; 3(3): 60-64.

30- De Giorgio R, Barbara G, Stanghellini V, et al: The pharmacological treatment of acute colonic pseudo-obstruction. Alimen Pharm \& Therap 2001; 15(11): 1717-1727.

31- Kukora JS, Dent TL: Colonoscopic decompression of massive nonobstructive cecal dilation. Arch Surg 1977; 112(4): 512-517.

32- Wegener M, Börsch G: Acute colonic pseudo-obstruction (Ogilvie's syndrome). Presentation of 14 of our own cases and analysis of 1027 cases reported in the literature. Surg Endosc 1987; 1(3): 169-174.

33- Harrison ME, Anderson MA, Appalaneni $\mathrm{V}$, et al: The role of endoscopy in the management of patients with known and suspected colonic obstruction and pseudoobstruction. GastrointestEndosc 2010; 71(4): 669-679.

34- Geller A, Petersen BT, Gostout CJ: Endoscopic decompression for acute colonic pseudo-obstruction. Gastrointest Endosc 1996; 44(2): 144-150.

35- Benacci JC, Wolff BG: Cecostomy: Therapeutic indications and results. Dis Colon Rectum 1995; 38: 530-534.

36- Casola G, Withers C, van Sonnenberg E, et al: Percutaneous cecostomy for decompression of the massively distended cecum. Radiology 1986; 58: 793-794. 